\title{
CARBON-CONSTRAINED PERISHABLE INVENTORY MANAGEMENT WITH FRESHNESS-DEPENDENT DEMAND
}

\author{
Hua, G. W. ."\#; Cheng, T. C. E. ${ }^{* *}$; Zhang, Y.*; Zhang, J. L.* \& Wang, S. Y.** \\ *School of Economics and Management, Beijing Jiaotong University, Beijing 100044, China \\ ** Department of Logistics and Maritime Studies, The Hong Kong Polytechnic University, \\ Hung Hom, Kowloon, Hong Kong, China \\ Academy of Mathematics and Systems Science, Chinese Academy of Sciences, \\ Beijing, 100190, China \\ E-Mail: huagw@amss.ac.cn ( ${ }^{\#}$ Corresponding author)
}

\begin{abstract}
We consider perishable inventory control with freshness-dependent demand under carbon emissions constraints. We propose two deteriorating inventory models with carbon emissions tax and the capand-trade mechanism, in which the demand is freshness dependent, carbon emissions come from inventory holding, shipping, and item deteriorating, and the objective is to maximize the profit per unit time. We characterize the existence and uniqueness of the solutions for the models. We analyse the impacts of carbon emissions tax, carbon emissions quota, and carbon price on inventory decisions, carbon emissions, and profit. We conduct simulation to generate managerial insights from our analytical results.

(Received, processed, and accepted by the Chinese Representative Office.)
\end{abstract}

Key Words: Deteriorating Inventory, Carbon Emissions Tax, Cap-and-Trade, Perishable Items, Freshness-Dependent Demand

\section{INTRODUCTION}

It is widely agreed that firms should take measures to reduce their carbon emissions to prevent global warming from worsening. To this end, governments have designed mechanisms to induce firms to reduce carbon emissions, such as mandatory regulations, carbon emissions tax, cap-and-trade, carbon offset etc. Under such circumstances, firms have to optimize their strategic or operational decisions concerning their supply chains, and their production, logistics, and inventory activities, to cope with the government initiatives.

There are studies on inventory management under carbon emissions constraints [1-6]. However, to the best of our knowledge, the extant literature has not considered one important class of inventory management, i.e., perishable inventory management. We fill this research gap by considering perishable inventory management with freshness-dependent demand under carbon emissions constraints. We focus on two popular kinds of carbon emissions constraints, namely carbon emissions tax and cap-and-trade regulation. Presenting two perishable inventory models corresponding to these two settings, we characterize the existence and uniqueness of the solutions for the models, and analyse the impacts of carbon emissions tax, carbon price, and carbon emissions quota on inventory decisions, carbon emissions, and profit. Given perishable inventory issues are very difficult to address analytically, our problem with freshness-dependent demand and carbon constraints is even harder to deal with, so we resort to simulation to complement the analytical analysis.

Our research is related to perishable inventory management, carbon-constrained inventory management, and issues associated with freshness-dependent demand. In the following we provide a short review of these three streams of research.

A perishable item has constant utility before its expiration date, after which the utility drops sharply, even to zero. Many products such as drugs, human blood, milk, meat, 
vegetables, fruits, and some electronic products are perishable items, which have certain or uncertain product lives. In [7-11] the authors provided reviews of inventory control of perishable items. There is a large body of literature on this topic, which extends the classical perishable model or addresses the issues in the context of supply chains. Time-dependent backlogging rate to extend the model suggested by Padmanabhan and Vrat is incorporated in [12]. How to manage slow-moving perishable products with expiration dates and fixed lot sizes is examined in [13]. In [14] is developed a model for perishable items with partial backlogging rates and freshness-dependent demand. The authors of [15] extended the model in [16] to incorporate item quality information into the perishable inventory model, where the demand depends on the item's instantaneous quality and the amount of shelf-space allocated to it. Since most deteriorating items are characteristic of dual uncertainty, i.e., demand and deterioration, in [17] is developed a newsvendor model for a deteriorating item with Poissontype deterioration and lognormal demand. Multi-objective mixed-integer linear programming models for perishable food supply chains are developed in [18]. In [19] are presented approximation algorithms for perishable inventory models with backlogging and lost-sales.

The second stream of related research is inventory management under carbon constraints. Pioneered research on this topic is included in $[1,3,20]$. In $[3,21]$ is showed that greenhouse gas emissions can be reduced through operations management. The authors of $[1,20]$ incorporated the carbon emissions trading mechanism into the EOQ model to decide the ordering size and selling price, and examined the impacts of carbon trade on order decisions, carbon emissions, and total cost. Carbon constraints are incorporated into the EOQ model [4] and showed that carbon emissions can be significantly reduced without significantly increasing cost via operational adjustments alone. In [22] the carbon emissions constraints are introduced to the multi-sourcing lot-sizing problem. They examined the uncapacitated lotsizing problem under four types of constraints. While in $[2,6,23]$ the carbon constraints are incorporated into the newsvendor model, in [24, 25] are incorporated sustainability concerns into the EOQ model and re-formulated the problem as a multi-objective problem. The authors of [5] developed a profit-maximization model for the production planning problem with the cap-and-trade.

The last stream of related research concerns freshness-dependent demand. In [16] is proposed a shelf-space-allocation and inventory model for fresh produce, whose demand depends on the items' freshness conditions and the displayed inventory. The supply chain of a fresh product in which the distributor can preserve the freshness of the product during its transportation is considered in [26]. The demand depends on the freshness-keeping effort. In [15] is examined perishable inventory problem with freshness and shelf-space-dependent demand. Inventory and shelf-space decisions with freshness and stock-dependent demand are investigated in [27].

We organize the rest of the paper organized as follows: In Section 2 we introduce the notation and assumptions used in the paper. In section 3 we present two deteriorating inventory models with carbon emissions tax and the cap-and-trade, and characterize the existence and uniqueness of the solutions for the models. In Section 4 we analyse the impacts of carbon emissions tax, carbon price, and carbon emissions quota on inventory decisions, carbon emissions, and profit. In Section 5 we present numerical simulation to generate practical insights form the analytical results. Finally, we conclude the paper and suggest future research topics in Section 6.

\section{ASSUMPTIONS AND NOTATION}

We define the notation and introduce the assumptions made in the paper as follows.

\section{Notation:}


- $t$ - time;

- $K$ - the fixed ordering cost per order, which is constant and known;

- $t_{0}$ - the time up to which inventory is positive in a cycle, a decision variable;

- $T$ - the cycle time, $T \geq t_{0}$, a decision variable;

- $C$ - the marginal replenishment cost per unit;

- $p$ - the selling price per unit, which is known and constant;

- $h$ - the inventory holding cost per unit per unit time, which is known and constant;

- $\theta$ - the constant deterioration rate;

- $I(t)$ - the inventory level on hand at time $t \in\left[0, t_{0}\right)$;

- $Q\left(t_{0}, T\right)$ - the order quantity in a cycle;

- $B\left(t_{0}, T\right)$ - the backlogging in a cycle;

- $D(t)$ - the demand rate function;

- $C_{1}$ - shortage cost per unit per unit time;

- $\Delta$ - carbon emissions quota per unit time;

- $P^{C}$ - carbon price per unit;

- $\tau$ - the carbon tax charged for per unit (ton);

- $P T\left(t_{0}, T\right)$ - the profit per unit time with carbon emissions tax;

- $P C\left(t_{0}, T\right)$ - the profit per unit time with cap-and-trade.

\section{Assumptions:}

1) A single deteriorating item is replenished, stored, and sold.

2) The replenishment rate is infinite with a zero lead-time.

3) Unsatisfied demand is fully backlogged.

4) The distribution of time to deterioration of the item follows the exponential distribution with parameter $\theta$.

5) The demand rate $D(t), t \in\left[0, t_{0}\right)$ is freshness-dependent

$$
D(t)= \begin{cases}\alpha e^{-\lambda t}, & 0 \leq t<t_{0}, \\ \alpha, & t_{0} \leq t<T .\end{cases}
$$

where $\alpha$ and $\lambda$ are positive constants and known, $e^{-\lambda t}$ is the freshness level of the item at time $t$, and $\lambda$ is the freshness factor.

6) The shipping cost is $T^{C}=u+v Q$ when the shipping size is $Q$ units, where $u$ and $v$ are positive constants and known.

7) Carbon emissions come from shipping, inventory holding, and item deteriorating.

8) The carbon emissions in executing an order of $Q$ units is linear in $Q$, i.e., $e_{0}+e Q$, where $e$ is the variable emissions factor.

9) The carbon emissions for holding $Q$ units of the item is linear in $Q$, i.e., $g_{0}+g Q$, where $g$ is the variable emissions factor in the warehouse.

10) The deteriorated item will emit carbon dioxide during deteriorating and the amount of carbon emissions per deteriorated item is $\gamma$.

\section{THE MODELS}

Based on the above notation and assumptions, we see that the item inventory decreases under the combined effects of demand and deterioration in $\left[0, t_{0}\right)$ and the demand backlogged in $\left[t_{0}, T\right)$. Then, we have:

$$
\frac{\mathrm{d} I(t)}{d t}= \begin{cases}\alpha e^{-\lambda t}-\theta I(t), & 0 \leq t<t_{0}, \\ -\alpha, & t_{0} \leq t<T .\end{cases}
$$

with the boundary condition $I\left(t_{0}\right)=0$. The solution of Eq. (2) is: 
1) when $\theta=\lambda$,

$$
I(t)= \begin{cases}\alpha\left(t_{0}-t\right) e^{-\theta t}, & 0 \leq t<t_{0}, \\ \alpha\left(t_{0}-t\right), & t_{0} \leq t<T .\end{cases}
$$

2) when $\theta \neq \lambda$,

$$
I(t)= \begin{cases}\frac{\alpha}{\theta-\lambda}\left[e^{(\theta-\lambda) t_{0}-\theta t}-e^{-\lambda t}\right], & 0 \leq t<t_{0}, \\ \alpha\left(t_{0}-t\right), & t_{0} \leq t<T .\end{cases}
$$

When $\lambda \rightarrow \theta, I(t)$ in Eq. (4) becomes that in Eq. (3). In other words, the case where $\lambda=\theta$ is a special case of $\lambda \neq \theta$. So, we mainly focus on the case where $\lambda \neq \theta$ in this paper.

We can obtain the following important formulas:

- the backlogging is $B\left(t_{0}, T\right)=\int_{t_{0}}^{T} \alpha d t=\alpha\left(T-t_{0}\right)$;

- the order quantity is $Q\left(t_{0}, T\right)=I(0)+B\left(t_{0}, T\right)=\frac{\alpha}{\theta-\lambda}\left[e^{(\theta-\lambda) t_{0}}-1\right]+\alpha\left(T-t_{0}\right)$;

- the total inventory is $T^{I}=\int_{0}^{t_{0}} I(t) d t=\frac{\alpha}{\theta(\theta-\lambda)} e^{(\theta-\lambda) t_{0}}+\frac{\alpha}{\lambda \theta} e^{-\lambda t_{0}}-\frac{\alpha}{\lambda(\theta-\lambda)}$;

- the amount of deteriorated items is $D^{I}=\int_{0}^{t_{0}} \theta I(t) d t=\frac{\alpha}{\theta-\lambda} e^{(\theta-\lambda) t_{0}}+\frac{\alpha}{\lambda} e^{-\lambda t_{0}}-\frac{\alpha \theta}{\lambda(\theta-\lambda)}$;

- the carbon emissions in shipping, inventory, and deteriorating items is

$$
\begin{aligned}
C^{E} & =e_{0}+e Q\left(t_{0}, T\right)+g_{0}+g T^{I}+\gamma D^{I} \\
& =\frac{\alpha(g+e \theta+\gamma \theta)}{\theta(\theta-\lambda)} e^{(\theta-\lambda) t_{0}}+\frac{\alpha(g+\gamma \theta)}{\lambda \theta} e^{-\lambda t_{0}}+\alpha e\left(T-t_{0}\right)+e_{0}+g_{0}-\frac{\alpha(g+e \lambda+\gamma \theta)}{\lambda(\theta-\lambda)} .
\end{aligned}
$$

Next, we present our profit-maximization model with carbon trade based on the above results. Our profit function consists of the following elements:

1) Ordering cost per cycle is $K$.

2) Holding cost per cycle is $H^{C}=h C T^{I}=\frac{\alpha h C}{\theta(\theta-\lambda)} \mathrm{e}^{(\theta-\lambda) t_{0}}+\frac{\alpha h C}{\lambda \theta} \mathrm{e}^{-\lambda t_{0}}-\frac{\alpha h C}{\lambda(\theta-\lambda)}$.

3) Shortage cost per cycle is $S^{C}=\frac{\alpha}{2} C_{1}\left(T-t_{0}\right)^{2}$.

4) Purchase cost per cycle is $P^{C}=C Q\left(t_{0}, T\right)=\frac{\alpha C}{\theta-\lambda} e^{(\theta-\lambda) t_{0}}-\frac{\alpha C}{\theta-\lambda}+\alpha C\left(T-t_{0}\right)$.

5) Shipping cost per cycle is $T^{C}=u+v Q\left(t_{0}, T\right)=\frac{\alpha \nu}{\theta-\lambda} e^{(\theta-\lambda) t_{0}}-\frac{\alpha \nu}{\theta-\lambda}+\alpha v\left(T-t_{0}\right)+u$.

6) The cost of carbon emissions in shipping, inventory holding, and item deterioration under carbon emissions tax is $T \alpha x^{C}=\tau C^{E}$.

7) The cost or revenue of carbon emissions in shipping, inventory holding and item deterioration under cap-and-trade is $\operatorname{Cap}^{C}=p^{C}\left(C^{E}-\Delta\right)$.

8) Sales revenue per cycle is $R=p\left(\int_{0}^{t_{0}} \alpha e^{-\lambda t} d t+\int_{t_{0}}^{T} \alpha d t\right)=\frac{\alpha p}{\lambda}\left(1-e^{-\lambda t_{0}}\right)+\alpha p\left(T-t_{0}\right)$.

\subsection{Carbon emissions tax case}

Given the above mentioned costs and revenue, the profit per unit time of the model is: 


$$
\begin{aligned}
P T\left(t_{0}, T\right) & =\frac{1}{T}\left\{R-K-H^{C}-S^{C}-P^{C}-T^{C}-T \alpha x^{C}\right\} \\
& =\frac{1}{T}\left\{A_{1} e^{(\theta-\lambda) t_{0}}+A_{2} e^{-\lambda t_{0}}-\frac{\alpha}{2} C_{1}\left(T-t_{0}\right)^{2}+A_{3}\left(T-t_{0}\right)+A_{0}\right\},
\end{aligned}
$$

where $A_{1}=-\frac{\alpha[h C+\theta C+\theta v+\tau(g+e \theta+\gamma \theta)]}{\theta(\theta-\lambda)}, A_{2}=-\frac{\alpha[p \theta+h C+\tau(g+\gamma \theta)]}{\lambda \theta}$,

$$
A_{3}=\alpha(p-C-v-\tau e), A_{0}=\frac{\alpha[p \theta+C h+\lambda(C+v-p)-\tau(g+e \lambda+\lambda \theta)]}{\lambda(\theta-\lambda)}-\tau\left(e_{0}+g_{0}\right)-K-u \text {. }
$$

Obviously, $p-C-v-\tau e>0$, i.e., the selling price is higher than the cost, comprising the purchase cost, the shipping cost, and the carbon emissions tax per unit item, which is reasonable. Then, we have $(\theta-\lambda) A_{1}<0, A_{2}<0, A_{3}>0$, and $(\lambda-\theta) A_{1}+\lambda A_{2}+A_{3}=0$.

Our Model 1 is $\max _{0 \leq t_{0} \leq T} P T\left(t_{0}, T\right)$. The necessary conditions to solve it are:

$$
\begin{gathered}
\frac{\partial P T\left(t_{0}, T\right)}{\partial t_{0}}=\frac{1}{T}\left\{(\theta-\lambda) A_{1} e^{(\theta-\lambda) t_{0}}-\lambda A_{2} e^{-\lambda t_{0}}+\alpha C_{1}\left(T-t_{0}\right)-A_{3}\right\}=0, \\
\frac{\partial P T\left(t_{0}, T\right)}{\partial T}=-\frac{1}{T^{2}}\left\{A_{1} e^{(\theta-\lambda) t_{0}}+A_{2} e^{-\lambda t_{0}}+\frac{\alpha}{2} C_{1}\left(T^{2}-t_{0}^{2}\right)-A_{3} t_{0}+A_{0}\right\}=0 .
\end{gathered}
$$

Re-arranging the terms in Eqs. (5) and (6), we have:

$$
\begin{gathered}
f^{2}\left(t_{0}\right)+2 \alpha C_{1}\left[\left(1+\lambda t_{0}-\theta t_{0}\right) A_{1} e^{(\theta-\lambda) t_{0}}+\left(1+\lambda t_{0}\right) A_{2} e^{-\lambda t_{0}}+A_{0}\right]=0, \\
T=t_{0}+\frac{f\left(t_{0}\right)}{\alpha C_{1}} .
\end{gathered}
$$

where $f\left(t_{0}\right)=(\lambda-\theta) A_{1} e^{(\theta-\lambda) t_{0}}+\lambda A_{2} e^{-\lambda t_{0}}+A_{3}$.

From Eqs. (6) and (8), we have:

$$
P T\left(t_{0}, T\right)=A_{3}-\alpha C_{1}\left(T-t_{0}\right)=A_{3}-f\left(t_{0}\right)=(\theta-\lambda) A_{1} e^{(\theta-\lambda) t_{0}}-\lambda A_{2} e^{-\lambda t_{0}} .
$$

Since $P T\left(t_{0}, \mathrm{~T}\right)>0,(\theta-\lambda) A_{1} e^{\theta t_{0}}>\lambda A_{2}$ and $t_{0}<\frac{1}{\theta} \ln \frac{-\lambda A_{2}}{(\lambda-\theta) A_{1}}=\bar{t}_{0}$. Noting that $-\lambda A_{2}-(\lambda-\theta) A_{1}=\frac{\alpha}{\theta}(p-C-v-\tau e)>0$, and $\overline{t_{0}}>0$, we have $p-C-v-\tau e-\frac{\theta}{\alpha}>0$.

Without loss of generality, we further suppose that $\max _{t_{0} \in\left[0, \tilde{t}_{0}\right]} P T\left(t_{0}, t_{0}\right)=P T\left(\tilde{t}_{0}, \tilde{t}_{0}\right)=\frac{1}{\tilde{t}_{0}}\left\{A_{1} e^{(\theta-\lambda) \tilde{t}_{0}}+A_{2} e^{-\lambda \tilde{t}_{0}}+A_{0}\right\}>0$, i.e., the maximum profit per unit time is positive when shortage is not allowed, which is reasonable.

Let $g\left(t_{0}\right)=f^{2}\left(t_{0}\right)+2 \alpha C_{1}\left[\left(1+\lambda t_{0}-\theta t_{0}\right) A_{1} e^{(\theta-\lambda) t_{0}}+\left(1+\lambda t_{0}\right) A_{2} e^{-\lambda t_{0}}+A_{0}\right]$, then $g\left(\overline{t_{0}}\right)=A_{3}^{2}+2 \alpha C_{1}\left[A_{1} e^{(\theta-\lambda) \bar{t}_{0}}+A_{2} e^{-\lambda \bar{t}_{0}}+A_{0}\right]$.

Since $\left[A_{1} e^{(\theta-\lambda) t_{0}}+A_{2} e^{-\lambda t_{0}}\right]^{\prime}=(\theta-\lambda) A_{1} e^{\theta t_{0}} e^{-\lambda t_{0}}-\lambda A_{2} e^{-\lambda t_{0}}>0$, $g\left(\overline{t_{0}}\right)>A_{3}^{2}+2 \alpha C_{1}\left[A_{1} e^{(\theta-\lambda) \tilde{t}_{0}}+A_{2} e^{-\lambda \tilde{t}_{0}}+A_{0}\right]>0$.

From the above analysis, we have the following proposition for the model. 


\section{Proposition 1.}

(1) If $A_{0}+A_{1}+A_{2} \leq 0$, then the solution $\left(t_{0}^{*}, T^{*}\right), t_{0}^{*} \in\left(0, \bar{t}_{0}\right)$, that solves Eqs. (7) and (8) simultaneously exists and is unique.

(2) If $A_{0}+A_{1}+A_{2}>0$, then the optimal solution for Model 1does not exist.

Based on the above analysis, we have the following proposition, which indicates that Model 1 has a unique optimal solution if it has a solution.

Proposition 2. The solution $\left(t_{0}^{*}, T^{*}\right)$ that solves Eqs. (7) and (8) is the unique optimal solution for Model 1.

Propositions 1 and 2 indicate that $A_{0}+A_{1}+A_{2}$ is the discriminant of Model 1: if $A_{0}+A_{1}+A_{2} \leq 0$, the solution of Model 1 exists and is unique; if $A_{0}+A_{1}+A_{2}>0$, the solution of Model 1 does not exist. From Eq. (7) and Proposition 2, we have:

$\left(1+\lambda t_{0}^{*}-\theta t_{0}^{*}\right) A_{1} e^{(\theta-\lambda) t_{0}^{*}}+\left(1+\lambda t_{0}^{*}\right) A_{2} e^{-\lambda t_{0}^{*}}+A_{0}<0$.

\subsection{Cap-and-trade case}

The profit per unit time for this case is

$$
\begin{gathered}
P C\left(t_{0}, T\right)=\frac{1}{T}\left\{R-K-H^{C}-S^{C}-P^{C}-T^{C}-C^{C} p^{C}\right\} \\
=\frac{1}{T}\left\{B_{1} e^{(\theta-\lambda) t_{0}}+B_{2} e^{-\lambda t_{0}}-\frac{\alpha}{2} C_{1}\left(T-t_{0}\right)^{2}+B_{3}\left(T-t_{0}\right)+B_{0}\right\}, \\
\text { where } B_{1}=-\frac{\alpha\left[C h+C \theta+\theta v+p^{C}(g+e \theta+\gamma \theta)\right]}{\theta(\theta-\lambda)}, B_{2}=-\frac{\alpha\left[p \theta+C h+p^{C}(g+\gamma \theta)\right]}{\lambda \theta}, \\
B_{3}=\alpha\left(p-C-v-p^{C} e\right), \\
B_{0}=\frac{\alpha\left[p \theta+h C+\lambda(C+v-p)-p^{C}(g+e \lambda+\lambda \theta)\right]}{\lambda(\theta-\lambda)}+p^{C}\left(\Delta-e_{0}-g_{0}\right)-K-u .
\end{gathered}
$$

We suppose that $p-C-v-p^{C} e>0$, i.e., the selling price is higher than the cost, comprising the purchase cost, the shipping cost, and the carbon emissions cost per unit item. Similar to Model 1, we have $(\theta-\lambda) B_{1}<0, B_{2}<0, B_{3}>0$, and $(\lambda-\theta) B_{1}+\lambda B_{2}+B_{3}=0$.

Our Model 2 is $\max _{0 \leq t_{0} \leq T} P T\left(t_{0}, T\right)$. The necessary conditions to solve it are:

$$
\begin{gathered}
h\left(t_{0}\right)^{2}+2 \alpha C_{1}\left[\left(1+\lambda t_{0}-\theta t_{0}\right) B_{1} e^{(\theta-\lambda) t_{0}}+\left(1+\lambda t_{0}\right) B_{2} e^{-\lambda t_{0}}+B_{0}\right]=0, \\
T=t_{0}+\frac{1}{\alpha C_{1}}\left[(\lambda-\theta) B_{1} e^{(\theta-\lambda) t_{0}}+\lambda B_{2} e^{-\lambda t_{0}}+B_{3}\right],
\end{gathered}
$$

where $h\left(t_{0}\right)=(\lambda-\theta) B_{1} e^{(\theta-\lambda) t_{0}}+\lambda B_{2} e^{-\lambda t_{0}}+B_{3}$. Similar to Model 1 , we also have $P C\left(t_{0}, T\right)=(\theta-\lambda) B_{1} e^{(\theta-\lambda) t_{0}}-\lambda B_{2} e^{-\lambda t_{0}}$. Letting $\hat{t}_{0}=\frac{1}{\theta} \ln \frac{-\lambda B_{2}}{(\lambda-\theta) B_{1}}$, similar to the carbon emissions tax case, we have the following results.

\section{Proposition 3.}

(1) If $B_{0}+B_{1}+B_{2} \leq 0$, then the solution $\left(t_{0}^{*}, T^{*}\right), t_{0}^{*} \in\left(0, \hat{t}_{0}\right)$, that solves Eqs. (10) and (11) simultaneously exists and is unique.

(2) If $B_{0}+B_{1}+B_{2}>0$, then the optimal solution for Model 2 does not exist. 


\section{Proposition 4.}

The solution $\left(t_{0}^{*}, T^{*}\right)$ that solves Eqs. (10) and (11) is the unique optimal solution for Model 2.

Similar to Model 1, Propositions 3 and 4 indicate that $B_{0}+B_{1}+B_{2}$ is the discriminant of Model 2: if $B_{0}+B_{1}+B_{2} \leq 0$, the solution of Model 2 exists and is unique; if $B_{0}+B_{1}+B_{2}>0$, the solution of Model 2 does not exist.

Propositions 1 and 3 state the conditions under which the solutions for Model 1 and 2 exist, which indicate that the solution exists in most cases. Propositions 2 and 4 state that the solutions for the models are unique if they exist. From Eq. (10), we have: $\left(1+\lambda t_{0}^{*}-\theta t_{0}^{*}\right) B_{1} e^{(\theta-\lambda) t_{0}^{*}}+\left(1+\lambda t_{0}^{*}\right) B_{2} e^{-\lambda t_{0}^{*}}+B_{0}<0$.

\section{THE IMPACT OF CARBON CONSTRAINT ON THE DETERIORATING INVENTORY STRATEGY}

In this section we examine the impacts of carbon emissions tax, carbon price, and carbon emissions quota on inventory decisions, carbon emissions, and profit for the two cases.

\subsection{Carbon emissions tax case}

From Eq. (7), we have $\frac{d t_{0}}{d \tau}=\frac{\varphi\left(t_{0}\right)}{g^{\prime}\left(t_{0}\right)}$, where:

$$
\begin{aligned}
& \varphi\left(t_{0}\right)=\frac{2 \alpha}{\theta} e^{-\lambda t_{0}}\left\{(g+\gamma \theta)\left[f\left(t_{0}\right)+\alpha C_{1} t_{0}+\frac{\alpha C_{1}}{\lambda}\right]-(g+e \theta+\gamma \theta)\left[f\left(t_{0}\right)+\alpha C_{1} t_{0}+\frac{\alpha C_{1}}{\lambda-\theta}\right] e^{\theta t_{0}}\right\} \\
& +2 \alpha e f\left(t_{0}\right)+2 \alpha C_{1}\left[\frac{\alpha(g+e \lambda+\theta \lambda)}{\lambda(\theta-\lambda)}+e_{0}+g_{0}\right] .
\end{aligned}
$$

The following result summarizes the impacts of carbon tax on the decision variable $t_{0}$.

\section{Proposition 5.}

(1) If $\theta>\lambda$, when $t_{0}^{*}<t_{0}, \frac{d t_{0}^{*}}{d \tau}>0$; when $t_{0}^{*}>t_{0}, \frac{d t_{0}^{*}}{d \tau}<0$.

(2) If $\theta<\lambda$, then $\varphi\left(t_{0}^{*}\right) \frac{d t_{0}^{*}}{d \tau}>0$.

Proposition 5 states that when the deterioration rate is greater than the freshness factor, the inventory time $t_{0}$ first increases then decreases with increasing carbon tax, and when the deterioration rate is less than the freshness factor, whether the inventory time $t_{0}$ increases with increasing carbon tax depends only on the sign of $\varphi\left(t_{0}^{*}\right)$.

From Eqs. (7) and (8), we have the following results on the impacts of carbon tax on the order cycle time, order quantity, carbon emissions, and retailer's profit.

Proposition 6.

(1) $\psi\left(t_{0}^{*}\right) \frac{d T^{*}}{d \tau}>0$.

(2) $\phi\left(t_{0}^{*}\right) \frac{d Q\left(t_{0}^{*}, T_{0}^{*}\right)}{d \tau}>0$. 
(3) $\chi\left(t_{0}^{*}\right) \frac{d C^{E}}{d \tau}>0$.

(4) $\delta\left(t_{0}^{*}\right) \frac{d P T\left(t_{0}^{*}, T^{*}\right)}{d \tau}<0$.

In the above, $h\left(t_{0}\right)=(g+e \theta+\gamma \theta) e^{(\theta-\lambda) t_{0}}-(g+\gamma \theta) e^{-\lambda t_{0}}-e \theta$, $\psi\left(t_{0}\right)=\alpha h\left(t_{0}\right) g^{\prime}\left(t_{0}\right)+\theta\left[f^{\prime}\left(t_{0}\right)+\alpha C_{1}\right] \varphi\left(t_{0}\right)$, $\phi\left(t_{0}\right)=\alpha \theta C_{1} \varphi\left(t_{0}\right) e^{(\theta-\lambda) t_{0}}+\theta f^{\prime}\left(t_{0}\right) \varphi\left(t_{0}\right)+\alpha g^{\prime}\left(t_{0}\right) h\left(t_{0}\right)$, $\chi\left(t_{0}\right)=\alpha C_{1} \varphi\left(t_{0}\right) h\left(t_{0}\right)+e \theta\left[f^{\prime}\left(t_{0}\right)+\alpha C_{1}\right] \varphi\left(t_{0}\right)+\alpha e g^{\prime}\left(t_{0}\right) h\left(t_{0}\right)$, $\delta\left(t_{0}\right)=\frac{2 \alpha^{2} C_{1}}{\theta}\left[\frac{g+\gamma \theta}{\lambda} e^{-\lambda t_{0}}-\frac{g+e \theta+\gamma \theta}{\lambda-\theta} e^{(\theta-\lambda) t_{0}}\right]+2 \alpha e f\left(t_{0}\right)+2 \alpha C_{1}\left[\frac{\alpha(g+e \theta+\gamma \theta)}{\lambda(\theta-\lambda)}+e_{0}+g_{0}\right]$.

Proposition 6 states that whether the order cycle time, order quantity, carbon emissions, and retailer's profit increases with increasing the carbon tax only depend on $\psi\left(t_{0}^{*}\right), \phi\left(t_{0}^{*}\right)$, $\chi\left(t_{0}^{*}\right)$, and $\delta\left(t_{0}^{*}\right)$, respectively.

\subsection{Cap-and-trade case}

In the cap-and-trade case, we examine the impacts of carbon emissions quota and carbon price on the decision variables and the order quantity, carbon emissions, and total profit, which are summarized in the following result.

\section{Proposition 7.}

(1) $\frac{d t_{0}^{*}}{d \Delta}<0, \frac{d T^{*}}{d \Delta}<0$,

(2) $\frac{d Q\left(t_{0}^{*}, T_{0}^{*}\right)}{d \Delta}<0, \frac{d C^{E}\left(t_{0}^{*}, T_{0}^{*}\right)}{d \Delta}<0, \frac{d P C\left(t_{0}^{*}, T^{*}\right)}{d \Delta}>0$.

Proposition 7 states that the inventory time, order cycle time, order quantity, and carbon emissions decrease with increasing carbon emissions quota, and the retailer's profit increases with increasing carbon emissions quota, which is straightforward.

\section{SIMULATION RESULTS}

Since it is hard to analytically analyse the impacts of carbon price, carbon tax, and carbon emissions quota, we resort to numerical simulation to examine their impacts on the inventory time, order cycle time, order quantity, carbon emissions, and profit. We use Matlab, and Eqs. (10) and (11) to perform the simulation experiment.

We set the parameters as follows: $K=80, C=8, p=60, h=0.3, \alpha=6,000, \theta=0.06$, $C_{1}=4, \Delta=60, p^{C}=1, \lambda=0.04, u=10, v=0.1, e_{0}=10, e=1, g_{0}=5, g=1$, and $\gamma=0.2$. The simulation results are summarized in Tables I-III.

Table I shows the impacts of carbon tax on the inventory time, order quantity, order cycle time, carbon emissions, and profit. Specifically, the inventory time keeps constant, and the order cycle time, order quantity, and carbon emissions increase with increasing carbon tax, while the total profit decreases with increasing carbon tax. In fact, with increasing carbon tax, the retailer is induced to backlog more demand and satisfy demand late because there are no carbon emissions when backlogging, so the retailer could achieve scale economy in carbon 
emissions, which may result in more order quantity and more carbon emissions. Obviously, the profit will decrease because the related cost will increase with increasing carbon tax.

Table II summarizes the impacts of carbon emissions quota on the inventory time, order quantity, order cycle time, carbon emissions, and profit. Specifically, they keep constant with increasing carbon emissions quota, which are the same as the results in [1].

Table III summarizes the impacts of carbon price on the inventory time, order cycle time, order quantity, carbon emissions, and profit. Specifically, the inventory time remains constant, but the order cycle time, order quantity, and carbon emissions increase with increasing carbon price, and the profit decreases. These findings are very similar to those for the carbon tax case.

Table I: Impacts of carbon tax on inventory time, order cycle time, order quantity, carbon emissions, and profit.

\begin{tabular}{|c|c|c|c|c|c|}
\hline$\tau$ & 1 & 2 & 3 & 3.5 & 4 \\
\hline$t_{0}^{*}$ & 5.38 & 5.38 & 5.38 & 5.38 & 5.38 \\
\hline$T_{0}^{*}$ & 12.91 & 14.24 & 15.56 & 16.22 & 16.88 \\
\hline$Q\left(t_{0}^{*}, T_{0}^{*}\right)$ & 79270 & 87214 & 95158 & 99130 & 103100 \\
\hline$C^{E}\left(t_{0}^{*}, T_{0}^{*}\right)$ & 164270 & 172210 & 180160 & 184130 & 188100 \\
\hline$P C\left(t_{0}^{*}, T^{*}\right)$ & 124650 & 86879 & 49103 & 30216 & 11328 \\
\hline
\end{tabular}

Table II: Impacts of carbon emissions quota on inventory time, order cycle time, order quantity, carbon emissions, and profit.

\begin{tabular}{|c|c|c|c|c|c|}
\hline$\Delta$ & 20 & 60 & 100 & 200 & 600 \\
\hline$t_{0}^{*}$ & 5.38 & 5.38 & 5.38 & 5.38 & 5.38 \\
\hline$T_{0}^{*}$ & 12.91 & 12.91 & 12.91 & 12.91 & 12.91 \\
\hline$Q\left(t_{0}^{*}, T_{0}^{*}\right)$ & 79270 & 79270 & 79270 & 79270 & 79270 \\
\hline$C^{E}\left(t_{0}^{*}, T_{0}^{*}\right)$ & 164270 & 164270 & 164270 & 164270 & 164270 \\
\hline$P C\left(t_{0}^{*}, T^{*}\right)$ & 124650 & 124650 & 124650 & 124650 & 124650 \\
\hline
\end{tabular}

Table III: Impacts of carbon price on inventory time, order cycle time, order quantity, carbon emissions, and profit.

\begin{tabular}{|c|c|c|c|c|c|}
\hline$p^{C}$ & 0.5 & 1 & 2 & 3 & 4 \\
\hline$t_{0}^{*}$ & 5.38 & 5.38 & 5.38 & 5.38 & 5.38 \\
\hline$T_{0}^{*}$ & 12.25 & 12.91 & 14.24 & 15.56 & 16.88 \\
\hline$Q\left(t_{0}^{*}, T_{0}^{*}\right)$ & 75299 & 79270 & 87214 & 95158 & 103100 \\
\hline$C^{E}\left(t_{0}^{*}, T_{0}^{*}\right)$ & 160300 & 164270 & 172210 & 180160 & 188100 \\
\hline$P C\left(t_{0}^{*}, T^{*}\right)$ & 14354 & 124650 & 86879 & 49103 & 11328 \\
\hline
\end{tabular}




\section{CONCLUSIONS}

In this paper we consider deteriorating inventory models with freshness-dependent demand under carbon emissions constraints. We propose two new models with carbon emissions tax and the cap-and-trade, in which the demand is freshness-dependent, and carbon emissions come from inventory holding, shipping, and item deteriorating, and the model is to maximize the profit per unit time. We characterize the existence and uniqueness of the solutions for the proposed models. We analyse the impacts of carbon emissions tax, carbon price, and carbon emissions quota on inventory decisions, carbon emissions, and profit. We conduct numerical simulation to generate managerial insights from our analytical results.

We find that in general carbon tax and cap-and-trade have little impact on inventory time, but the order cycle time, order quantity, and carbon emissions will increase with increasing carbon price, and profit will decrease in the two cases since the two mechanisms will incur more cost.

Future research on this topic may examine the special case where the freshness factor has a deteriorating rate, which may yield a closed solution. Another research direction is to study the case with partial backlogging and lost sales.

\section{ACKNOWLEDGEMENTS}

This research was supported in part by the NSFC under grant numbers 71390334 and 71532002 , Beijing Philosophy and Social Science Foundation under grant number 13JGB042, and the Fundamental Research Funds for the Central Universities under grant number 2015 jbwy011.

\section{REFERENCES}

[1] Hua, G.-W.; Cheng, T. C. E.; Wang, S.-Y. (2011). Managing carbon footprints in inventory management, International Journal of Production Economics, Vol. 132, No. 2, 178-185, doi:10.1016/j.ijpe.2011.03.024

[2] Song, J.-P.; Leng, M.-M. (2012). Analysis of the single-period problem under carbon emission policies, Choi, T.-M. (Ed.), Handbook of Newsvendor Problems: Models, Extensions and Applications, Springer, New York, 297-313, doi:10.1007/978-1-4614-3600-3_13

[3] Benjaafar, S.; Li, Y.-Z.; Daskin, M. (2013). Carbon footprint and the management of supply chains: insights from simple models, IEEE Transactions on Automation Science and Engineering, Vol. 10, No. 1, 99-116, doi:10.1109/TASE.2012.2203304

[4] Chen, X.; Benjaafar, S.; Elomri, A. (2013). The carbon-constrained EOQ, Operations Research Letters, Vol. 41, No. 2, 172-179, doi:10.1016/j.orl.2012.12.003

[5] Zhang, B.; Xu, L. (2013). Multi-item production planning with carbon cap and trade mechanism, International Journal of Production Economics, Vol. 144, No. 1, 118-127, doi:10.1016/ j.ijpe.2013.01.024

[6] Liu, W.-H.; Xie, W.-C.; Hua, G.-W. (2013). Order decision with random demand: a research from the perspective of carbon emission cap and carbon trade mechanism, Chen, F.; Liu, Y.-S.; Hua, G.-W. (Eds.), LTLGB 2012 - Proceedings of International Conference on Low-carbon Transportation and Logistics, and Green Buildings, Springer-Verlag, Berlin, 585-593, doi:10.1007/978-3-642-34651-4_81

[7] Raafat, F. (1991). Survey of literature on continuously deteriorating inventory models, Journal of the Operational Research Society, Vol. 42, No. 1, 27-37, doi:10.1038/sj/jors/0420103

[8] Goyal, S. K.; Giri, B. C. (2001). Recent trends in modeling of deteriorating inventory, European Journal of Operational Research, Vol. 134, No. 1, 1-16, doi:10.1016/S0377-2217(00)00248-4

[9] Karaesmen, I. Z.; Scheller-Wolf, A.; Deniz, B. (2011). Managing perishable and aging inventories: review and future research directions, Kempf, K. G.; Keskinocak, P.; Uzsoy, R. (Eds.), Planning Production and Inventories in the Extended Enterprise, Springer, New York, 393-436, doi:10.1007/978-1-4419-6485-4_15 
[10] Nahmias, S. (2011). Perishable Inventory Systems, Springer, New York, doi:10.1007/978-14419-7999-5

[11] Bakker, M.; Riezebos, J.; Teunter, R. H. (2012). Review of inventory systems with deterioration since 2001, European Journal of Operational Research, Vol. 221, No. 2, 275-284, doi:10.1016/ j.ejor.2012.03.004

[12] Dye, C.-Y.; Ouyang, L.-Y. (2005). An EOQ model for perishable items under stock-dependent selling rate and time-dependent partial backlogging, European Journal of Operational Research, Vol. 163, No. 3, 776-783, doi:10.1016/j.ejor.2003.09.027

[13] Ketzenberg, M.; Ferguson, M. E. (2008). Managing slow-moving perishables in the grocery industry, Production and Operations Management, Vol. 17, No. 5, 513-521, doi:10.3401/ poms. 1080.0052

[14] Yan, X. (2012). An EOQ model for perishable items with freshness-dependent demand and partial backlogging, International Journal of Control and Automation, Vol. 5, No. 4, 19-38

[15] Piramuthu, S.; Zhou, W. (2013). RFID and perishable inventory management with shelf-space and freshness dependent demand, International Journal of Production Economics, Vol. 144, No. 2, 635-640, doi:10.1016/j.ijpe.2013.04.035

[16] Bai, R.-B.; Kendall, G. (2008). A model for fresh produce shelf-space allocation and inventory management with freshness-condition-dependent demand, INFORMS Journal on Computing, Vol. 20, No. 1, 78-85, doi:10.1287/ijoc.1070.0219

[17] Huang, M.-G. (2013). Economic ordering model for deteriorating items with random demand and deterioration, International Journal of Production Research, Vol. 51, No. 18, 5612-5624, doi: $10.1080 / 00207543.2013 .791753$

[18] Saengsathien, A. (2015). Modelling and determining inventory decisions for improved sustainability in perishable food supply chains, Dissertation Thesis, University of Exeter, Exeter

[19] Chao, X.; Gong, X.; Shi, C.; Zhang, H. (2015). Approximation algorithms for perishable inventory systems, Operations Research, Vol. 63, No. 3, 585-601, doi:10.1287/opre.2015.1386

[20] Hua, G.-W.; Qiao, H.; Li, J. (2011). Optimal order lot sizing and pricing with carbon trade, ICEIS 2011 - Proceedings of the $13^{\text {th }}$ International Conference on Enterprise Information Systems, 533-536, doi:10.2139/ssrn.1796507

[21] Plambeck, E. L. (2012). Reducing greenhouse gas emissions through operations and supply chain management, Energy Economics, Vol. 34, Supplement 1 (Green Perspectives), S64-S74, doi: $10.1016 /$ j.eneco.2012.08.031

[22] Absi, N.; Dauzère-Pérès, S.; Kedad-Sidhoum, S.; Penz, B.; Rapine, C. (2013). Lot sizing with carbon emission constraints, European Journal of Operational Research, Vol. 227, No. 1, 55-61, doi:10.1016/j.ejor.2012.11.044

[23] Rosič, H.; Jammernegg, W. (2013). The economic and environmental performance of dual sourcing: A newsvendor approach, International Journal of Production Economics, Vol. 143, No. 1, 109-119, doi:10.1016/j.ijpe.2012.12.007

[24] Bouchery, Y.; Ghaffari, A.; Jemai, Z.; Dallery, Y. (2012). Including sustainability criteria into inventory models, European Journal of Operational Research, Vol. 222, No. 2, 229-240, doi:10.1016/j.ejor.2012.05.004

[25] Bouchery, Y. (2012). Supply chain optimization with sustainability criteria: A focus on inventory models, Doctoral Thesis, Ecole Centrale Paris, Châtenay-Malabry

[26] Cai, X.-Q.; Chen, J.; Xiao, Y.-B.; Xu, X.-L. (2010). Optimization and coordination of fresh product supply chains with freshness-keeping effort, Production and Operations Management, Vol. 19, No. 3, 261-278, doi:10.1111/j.1937-5956.2009.01096.x

[27] Chen, S.-C.; Min, J.; Teng, J.-T.; Li, F. (2016). Inventory and shelf-space optimization for fresh produce with expiration date under freshness-and-stock-dependent demand rate, Journal of the Operational Research Society, Vol. 67, No. 6, 884-896, doi:10.1057/jors.2015.100

\section{APPENDIX}

All the proofs of the propositions can be found at: http://pan.baidu.com/s/lkVATZuv. 